\title{
COMPARISON AND VALIDATION OF WAKE VORTEX CHARACTERISTICS COLLECTED AT DIFFERENT AIRPORTS BY DIFFERENT SCANNING LIDAR SENSORS

\author{
Ludovic Thobois $^{1}$, Jean-Pierre Cariou ${ }^{1}$, Valerio Cappellazzo ${ }^{2}$, Christian Musson $^{2}$, Vincent Treve $^{2}$ \\ ${ }^{1}$ Leosphere, France, *lthobois@leosphere.com \\ ${ }^{2}$ EUROCONTROL, Belgium
}

\begin{abstract}
Today, the demand for increasing airport capacity is high, in particular for increasing runway throughput from an ATM perspective. Runway capacity is often directly linked with the minima longitudinal separation between aircraft on approach phase or between aircraft on departure. The separation minima are based on surveillance capabilities and on wake turbulence (WT) in order to mitigate respectively collision risk and WTinduced accidents, therefore WT hazard becomes a major concern for ATM. For ten years, many research LIDAR systems have been used for better understanding wake vortices behaviors in the operational environment within large range of wind and turbulence conditions. All these studies[1][2] helped to design new concepts of wake separations between aircrafts thanks to the proven capabilities of LIDAR systems to assess the risks of wake vortex (WV) encounters through the circulation retrievals. The re-categorization project, called RECAT [8], has been launched by a joint EUROCONTROL - FAA initiative in order to renew and optimize the out-of-date currently applied ICAO regulations on distance separation. Nowadays, the first phase of regional RECAT projects, which consists in defining new distance separation matrices composed of six/seven static aircraft categories instead of three, entered the operational phase and is deployed in several airports in United States and Europe. In addition, other concepts like Time-Based Separation have also been studied and deployed in London Heathrow. The airports where these solutions have been deployed obtained significant benefits as increased runway throughput and improved resilience to disruptions. For implementing such new WT solutions at an airport, a local safety assessment before the implementation and a risk monitoring after are usually needed. Before implementation, it may be required to determine for the targeted airport the relative variations of risk of wake vortex
\end{abstract}

encounters, given the local ATM rules, the traffic mix, the weather conditions and their impact on the wake vortex decay. After implementation, the risk monitoring might perform in-depth analysis of wake vortex encounter reported by pilots. For all the mentioned steps, the use of scanning Doppler LIDARs is the only experimental sensor capable of measuring the localization and the circulation of the wake vortices and to provide ground truth wake vortex measurements. Next generation operational LIDARs need to be developed to address in a cost effective way these operational needs. Furthermore, a specific configuration and methodology need to be developed to ensure the accuracy of the wake vortex data. Such a LIDAR based wake vortex solution has been tested at Paris Charles De Gaulle which implemented the RECAT-EU wake separation scheme. The wake vortex circulation, initial spacing and decay measured have been compared to the data collected in London Heathrow by a different LIDAR sensor. The results indicated that the initial circulation, the time to demise, the decay curve evolution and the vortex spacing are very coherent between the two databases.

\section{INTRODUCTION}

Wake vortices (WV) are a consequence of the pressure differential between the lower and upper surface of the wing moving through the air. The trailing vortices rolls-up into a pair of coherent, counter-rotating vortices that can persist for a considerable length of time after the aircraft has flown by and could pose a potential hazard to the safe flight of an aircraft that is following the lead aircraft and encountering these vortices. The trailing vortices' rate and time to dissipate (decay) depend on the weight, size, wing configuration and speed of the aircraft as well as atmospheric conditions. Specifically, the roll-up of two-vortex system (2VS) formed behind an aircraft is composed of two counter-rotating vortices of 
initial total circulation $\Gamma_{0}$ and initially spaced by a distance $b_{0}$ with $s$ the spacing factor that depends on the combined effects of wing and horizontal tail plane (HTP) loadings. The initial total circulation is related to the aircraft weight, $W_{l}$, flight speed $V_{l}$, wing span $b_{l}$ and wing and horizontal tail plane loadings:

$$
\Gamma_{0}=\frac{W_{l}}{\rho V_{l} b_{l} s}
$$

Where $\rho$ is the air density, the sub index $l$ refers to leader aircraft and $s$ is the spacing factor defined as the ratio between the initial lateral spacing between the vortices $b_{0}$ and the aircraft $\operatorname{span} b_{l}$. The vortex total circulation $\Gamma_{\text {tot }}$ decreases in time, starting from $\Gamma_{0}$ and following a two-phase decay, with a decay rate that depends on the atmospheric turbulence, characterized by the Eddy Dissipation Rate (EDR), $\epsilon$, and thermal stratification, characterized by the Brunt-Väisälä frequency[4], $N$. To measure properly $\mathrm{WV}$, scanning coherent Doppler Lidar (CDL) are used when configured with high resolution $(<10 \mathrm{~m})$ and high update rate (10s). Commonly, the experimental setup used is to perform a vertical slice perpendicular to the runway in order to characterize $\mathrm{WV}$ in a monitoring area of $500 \mathrm{~m}$ width and at an altitude of one wingspan. For optimizing the distance separations to be applied between aircrafts, huge wake vortex databases are required to be collected by CDLs. In this study, two types of CDLs have been used: the first CDL is a WindTracer with which a database has been collected a few years ago at Heathrow (EGLL-1) airport and the second CDL is a WIDCUBE200S which has been deployed at Charles De Gaulle (CDG) airport 1 year ago. The objective of this work is now to compare the two WV databases collected by two sensors with different WV characterization algorithms and at two airports with different atmospheric environments. Therefore it is of interest to assess the wake vortex characteristics recorded by both sensors in order to identify similarities and differences of the two campaigns. The comparison is focused on $\Gamma_{0}$ and $b_{0}$ measurements as they are important parameters that drive the entire WV physics as described above.

\section{METHODOLOGY}

\subsection{Wake vortex algorithms for CDLs}

Many studies show the capabilities to track WV within the Doppler spectra and also within the radial wind data [5][6][7]. In the past two years, WV algorithms have been developed based on the fitting of a WV theoretical model on radial wind speeds [7]. This method is used in the WINDCUBE WV characterization algorithm which detects, localizes and computes the circulation of WV. This last step is performed by a least mean square optimization of a HallockBurnham WV theoretical model on the radial wind speeds, as proposed by Smalikho [7]. This algorithm has been validated on real wake vortex data collected at Paris-Charles-De-Gaulle airport in 2011 and at Toulouse-Blagnac airport in 2014 [9].

\subsection{Two-Phase Decay curve}

All the measured data from the CDG and EGLL-1 databases have been used to reconstruct a generic (per aircraft type) two-phase decay curve that represents the WV physics. Each phase is analytically described by a decreasing exponential function characterized by coefficients $\alpha_{1}$ and $\alpha_{2}$ and with $\Gamma_{0}$ as initial circulation. The transition between the two phases occurs at $t=t_{c}$ which can be viewed as a measured time-to-demise (dashed lines in Figure 1 and Figure 2). The mathematical formalization of the fit $\Gamma^{*}(t)$ can be written as:

$$
\Gamma^{*}(t)= \begin{cases}\Gamma_{0} \exp \left(-\alpha_{1} t\right) & \text { for } t \leq t_{c} \\ \Gamma_{0} \exp \left(\alpha_{2} t_{c}-t\right)-\alpha_{1} t_{c} & \text { for } t>t_{c}\end{cases}
$$

Four parameters have to be fixed: $\Gamma_{0}, \alpha_{1}, \alpha_{2}$ and $t_{c}$. They are determined by minimizing residues between the fit $\Gamma^{*}(t)$ and the data $\Gamma(t)$. Residues are defined by the least squares:

$$
\text { res }=\sum_{i=1}^{N}\left(\Gamma^{*}\left(\alpha_{1}, \alpha_{2}, t_{c}, \Gamma_{0} ; t_{i}\right)-\Gamma\left(t_{i}\right)\right)^{2}
$$

With the use of numerical computing software, a (local) solution to this multi-variables minimization problem without constraint is found. In the fit no extrapolation of the lateral WV position has been considered; it conservatively assumed that the WV lateral positions after the last measured point remain constant.

\section{RESULTS}


The wake vortex algorithm has been applied to collect wake vortex data at Paris-Charles de Gaulle airport. The analyses are conducted for the CDG Database containing about 26000 tracks from 19th November 2015 to 22nd September 2016 with the Leosphere LiDAR and compared with the EGLL-1 Database containing about 55000 tracks collected from October 2008 until December 2010 where a different LIDAR has been used.

\subsection{Wake Vortex Circulation and Decay}

In Figure 1 and Figure 2 the comparison between the CDG Database (blue curve), and the EGLL-1 Database (red curve) with the two-phase decay curve are presented. The resulting plot is the mean of all the decay curves, in every plot there is the indication of total number of tracks in the database, the number of tracks taken in account by the method (between brackets) and the number of tracks that contains NaN values. As we can see in the results the initial vortex circulation $\Gamma_{0}$, the time to demise and the decay curve are very coherent between the two databases. This is valid for all the aircraft types where a sufficient number of tracks for the comparison are collected.

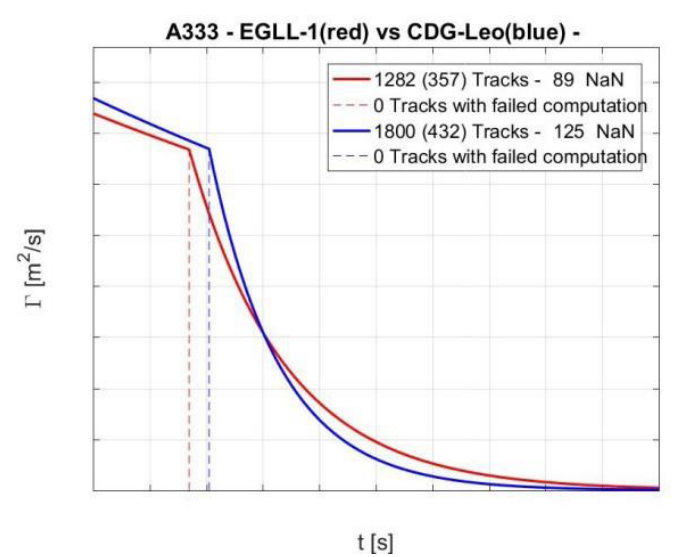

Figure 1: Wake decay comparison - A333

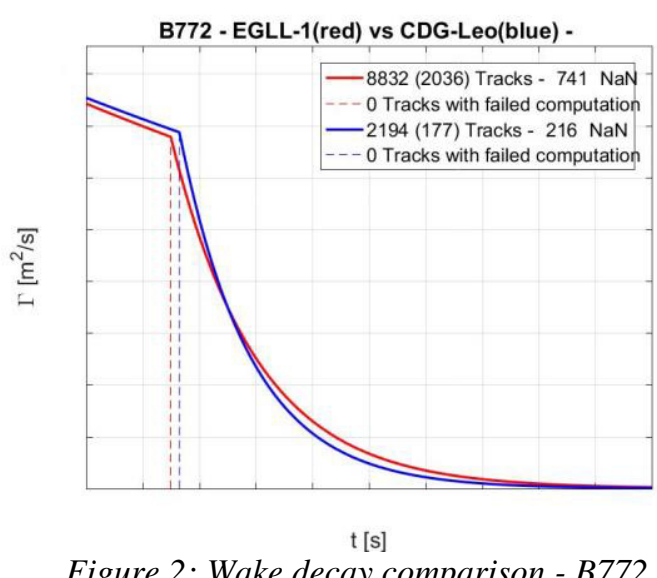

\subsection{Initial vortex spacing}

The data collected have also been analyzed to compare the initial vortex spacing $\left(b_{0}\right)$. The $b_{0}$ are obtained following the 2 steps methodology presented in the RECAT-EU Safety Case [8]. For step 1 of the $b_{0}$ determination process, the $b_{0}$ measured in London Heathrow (EGLL-1 database) are used as altitude threshold for the CDG measurement selection (avoiding an iterative process). The Step 2 is used for better characterization of the $b_{0}$ for all the aircraft that do not have the complete roll-up of the vortex at one wingspan altitude. The mean and the median values of $b_{0}$ are calculated using all selected tracks for each aircraft type. The CDG results are compared in Table 1 with the results of EUROCONTROL analysis of the EGLL-1 database.

The vortex spacing factor $s=b_{0} / b$ measured in CDG datasets are found to be, on average, between $2 \%$ and $14 \%$ bigger than those of the EGLL-1 dataset, however for many aircraft types the number of tracks collected that satisfies the altitude and time conditions identified by the methodology is limited. Some high differences like in the case of B752 could be justified by the different operating conditions at the two airports. The differences observed for the Boeing 777 family can be associated to a bad identification of the different versions B772 and B77W leading to an error of $7-11 \%$. Some measurements of $b_{0}$ are also available for the A388-800 (A388), the obtained values are found consistent (only $2.15 \%$ deviation) with the A388 EGLL-1 measurements. Then, only the aircraft for which about 100 tracks (or more) were collected and with no problems in 
the identification and operations were taken into account. Applying this methodology, the computed ratio between the b0 measured in EGLL-1 and the median b0 measured in CDG shown a systematic error on the b0 measurement of about $2 \%$ (see Table 2).

\begin{tabular}{|c|c|c|}
\hline AC & rel \% to EGLL & \# ac \\
\hline $\mathbf{A 3 3 2}$ & $0.38 \%$ & 336 \\
\hline $\mathbf{A 3 3 3}$ & $2.02 \%$ & 282 \\
\hline $\mathbf{A 3 4 3}$ & $-2.46 \%$ & 102 \\
\hline $\mathbf{A 3 8 8}$ & $-2.15 \%$ & 107 \\
\hline $\mathbf{B 7 4 4}$ & $-1.88 \%$ & 6 \\
\hline $\mathbf{B 7 5 2}$ & $-14.74 \%$ & 529 \\
\hline $\mathbf{B 7 6 3}$ & $-6.19 \%$ & 250 \\
\hline $\mathbf{B 7 7 2}$ & $-11.79 \%$ & 529 \\
\hline $\mathbf{B 7 7 W}$ & $-8.64 \%$ & 654 \\
\hline
\end{tabular}

Table 1 - b0 analysis of CDG Dataset

\begin{tabular}{|c|c|c|c|}
\hline AC & b0 Ratio (\%) & Wingspan & $\begin{array}{c}\text { Aircraft } \\
\text { Type }\end{array}$ \\
\hline A332 & 98 & 60.3 & H \\
\hline A333 & 97 & 60.3 & H \\
\hline A343 & 100 & 60.3 & $\mathrm{H}$ \\
\hline A388 & 96 & 79.75 & J \\
\hline B744 & 102 & 64.44 & $\mathrm{H}$ \\
\hline B752 & 111 & 38.05 & $\mathrm{M}$ \\
\hline B763 & 101 & 47.57 & $\mathrm{H}$ \\
\hline B772 & 111 & 60.93 & $\mathrm{H}$ \\
\hline B77W & 107 & 64.8 & $\mathrm{H}$ \\
\hline
\end{tabular}

Table 2 - bo Ratio

\section{CONCLUSIONS}

To sum up, the comparison of the two datasets show good agreements in terms of wake vortex decay, initial circulation, time to demise, and vortex initial spacing. For this last parameter, a mean error of about $2 \%$ has been obtained with a given methodology consisting in the sufficient data sampling for each aircraft type and in the filtering of the aircrafts that were badly identified or not operated in the same way at the two airports. Even if these data collections have been performed at different airports, by different Lidar sensors and despite the fact they have been analyzed by different wake vortex characterization algorithms, there is already a reliable degree of consistency between the two wake vortex datasets, which create a solid basis for the future work. The final goal is to have a robust methodology to build consistent and universal wake vortex datasets with different scanning LIDAR sensors. This way, all those datasets can be used in support of safety assessment necessary to deploy wake solutions that can tackle the airports capacity issues.

\section{ACKNOWLEDGEMENTS}

This paper is based on the LIDAR data campaign jointly conducted with NATS (in Heathrow) and DSNA (in Paris-Charles-de-Gaulle).

\section{References}

[1] CREDOS: Crosswind-Reduced Separations for Departure Operations, EU Project Program: FP6AEROSPACE, Ref.: 30837, Record Number: 81468.

[2] FAR WAKE Fundamental Research on Aircraft Wake Phenomena, EU project Funded under: FP6AEROSPACE Ref.: 12238, Start date: 2005-02-01, End date: 2008-05-31.

[3] Greene, G. C., "An Approximate model of vortex decay in atmosphere," J. Aircraft, Vol. 23, July 1986, pp. 566-573.

[4] De Visscher, I. et al., "Aircraft vortices in stably stratified and weakly turbulent atmo-spheres: simulation and modeling," AIAA J., Vol. 51, No. 3, March 2013, pp. 551-566.

[5] Köpp, F., et al. "Characterization of aircraft wake vortices by 2-micro pulsed doppler lidar", Journal of Atmospheric and Oceanic Technology, vol. 21, no. 2, pp. 194-206, 2004.

[6] Dolfi-Bouteyre, A., et al., "1.5 m all fiber pulsed lidar for wake vortex monitoring, Conference on Lasers and Electro-Optics", Optical Society of America, 2008.

[7] Smalikho, I.N.; Banakh, V.A.; Holzäpfel, F.; Rahm, S. Method of radial velocities for the estimation of aircraft wake vortex parameters from data measured by coherent Doppler lidar. 2015. Optics Express 23(19) A1194-A1207.

[8] EUROCONTROL, European Proposal for revised Wake Turbulence Categorisation and Separation Minima on Approach and Departure, "RECAT - EU", Safety Case Report, 2013.

[9] Thobois, L., et al. "Deployment of a next generation and operational LIDAR solution for monitoring wake vortices for supporting new wake turbulence regulations (Invited)", 8th AIAA Atmospheric and Space Environments Conference, AIAA Aviation, (AIAA 2016-3273) 